bearing on the etiology of XX males. Clin Genet 1977; 11:91-106.

12 Powers HO, Neu RL, Smulyan H, Gardner LI. An adult phenotypic male with a $46, X X$ chromosome complement. $J$ Clin Endocrinol Metab 1970;31:576-9.

13 Bartsch-Sandhoff M, Schade H, Wiegelmann W, Solbach H, Scholz W. Ein Beitrag zur Geneses von XX-Männer. Humangenetik $1974 ; 21: 245-53$.

14 Dosik H, Wachtel SS, Khan F, Spergel G, Koo GC. Y-chromosomal genes in a phenotypic male with a 46,XX karyotype. JAMA 1976;236:2505-8.

15 Mirò R, Caballin MR, Marina S, Egozcue J. Mosaicism in XX males. Hum Genet 1978;45:103-6.

16 Pawlowitzki JH, Holzgreve W, Kövary M, Niermann H, Scholz W. Testing mosaicism in a XX male. Ann Genet $1978 ; 21: 152-6$.

Requests for reprints to Dr U Bigozzi, Cattedra di Genetica Medica, Viale Morgagni 85, Firenze, Italy.

\section{Complex chromosomal rearrangement leading to partial trisomy 22}

SUMMARY We have examined a boy with a peculiar facial appearance and mental retardation. Cytogenetic studies showed 47,XY, monosomy 22 , + two marker chromosomes, M1 and M2. The karyotype is interpreted as functionally partial trisomy 22. Chromosome analyses of both parents and three sibs were normal.

Several cases of trisomy 22 (non-mongoloid trisomy G) have been described. ${ }^{1-5}$ There is a wide variation in the phenotypic appearance of these cases. However, there are many similarities which allow the description of a specific syndrome. It has been suggested by Zellweger et $a l^{5}$ that trisomy 22 syndrome (T22), cat eye syndrome (CES), which is a partial trisomy 22 , and cases with symptoms of both T22 and CES, the so-called intermediate cases, are variants of the same disorder. The main feature(s) of T22, cleft palate, and of CES, coloboma of the iris and imperforate anus, are absent in our patient. He has, however, some of the characteristics of T22 or CES or both, suggesting an intermediate case. The purpose of this report is to describe the clinical picture and the concomitant chromosomal aberrations.

\section{Case report}

The proband (fig 1) was born in January 1975 after an uneventful pregnancy; the mother was 24 and the



FIG 1 Front and side view of proband.

father 30 years old. The mother has two daughters born in 1972 and 1978, and one son born ir? 1973, all of whom are normal. The parents are़्र unrelated. The boy was small (birthweight $2780 \mathrm{~g}$, height $48 \mathrm{~cm}$, head circumference $34.5 \mathrm{~cm}$ ). He waso hypotonic and cyanotic after birth, needing oxygergo for 6 hours. Apgar score at 1 minute was 8 . Physical examination showed right-sided cryptorchidism. He had a peculiar facial appearance and poorly formed ears. Routine laboratory tests were normal.

He was admitted to the paediatric ward twice a the age of 2 and $4 \frac{1}{2}$ months because of continued vomiting, seborrhoeic dermatitis, and episodes of coughing and wheezing. He was thin and his peculiar appearance was now more marked with hyper telorism, antimongoloid slanting of the eyes, eyelid folds, epicanthus, and mild bilateral ptosis. He had micrognathia, large, low-set ears with an indentatioff on each ear lobe, and small bilateral preauriculas sinuses. There was frontal bossing, his nose was broad with a depressed nasal bridge, and the mouth? was downturned. He had low-set, widely spaced nipples, moderate cubitus valgus, and short lower extremities. His motor development was normal, but his mental milestones were delayed. The back of his: head was flat and asymmetrical. Laboratorys investigations, including urinary mucopolysac charides, liver function tests, thyroid function test repeated urinary amino-acid screening, and mals absorption tests, were normal. IgG, IgA, and IgMe were normal, but IgE was raised $(120 \mathrm{U} / \mathrm{ml})$. Radiog allergen sorbent test for egg albumin was positive $\mathbb{\complement}$ The leucocyte count was variable (35000 to 7400$\left.)^{\text {? }}\right)^{4}$ with marked and prolonged leucocytosis, but a bone marrow puncture was normal. Intravenous pyelo웅 gram, electroencephalogram, and chest $x$-ray were normal. A barium meal showed massive regurgita tion into the oesophagus when the patient was 
horizontal, which was taken as a sign of sphincter insufficiency.

At 14 months a bronchoscopy was done because of a constant cough and periodic respiratory difficulties. Chronic oesophagitis and diastema laryngis were found. At this time both testicles were palpable in the inguinal canal. Despite being kept in an upright position most of the time, there were long periods when he had considerable feeding difficulties during the following year.

He was last seen at the age of $3 \frac{1}{2}$ years. He was then an anxious boy with poor concentration. His motor development was normal, but mentally he was considerably retarded (IQ $\bumpeq 60$ ) and his speech was poor. His height was $84 \mathrm{~cm}(3 \mathrm{~cm}$ below $-3 \mathrm{SD})$ and his weight was $13 \mathrm{~kg}$ (between -1 and $-2 \mathrm{SD}$ ) which indicates growth retardation.

\section{CYTOGENETIC EXAMINATION}

Cytogenetic examination of the proband was performed on three different occasions with identical results using G-, C-, Q-, and R-banding and silver staining techniques. A modal count of 47 chromosomes was found in all 110 cells analysed. Only one normal chromosome 22 was seen. Two small chromosomes the size of the $\mathrm{G}$ group chromosomes were seen, M1 and M2 (fig 2). M1 was almost metacentric, but was definitely not an isochromosome as both arms appeared to be longer than 22p. M1 had satellites at both ends, with bipartite $C$ bands, but only one centromere. M2 was slightly submetacentric with satellites at the end of the longest arm, and also had a distal $\mathrm{C}$ band removed from the centromere region.

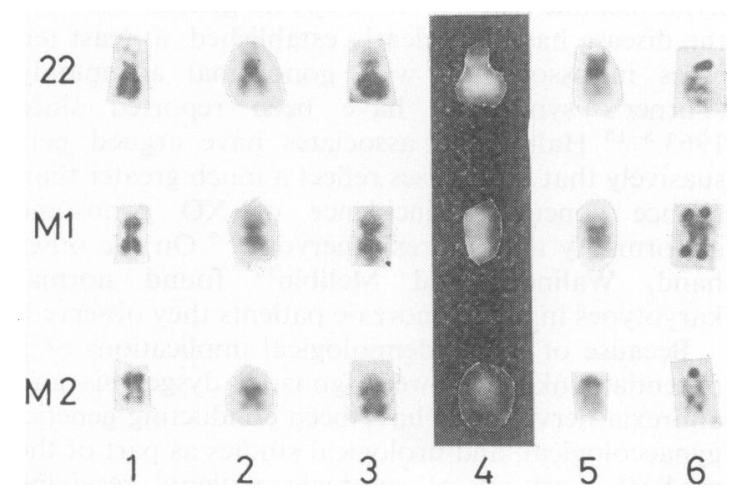

FIG 2 Partial karyotypes showing normal chromosome 22 and marker chromosomes M1 and M2, stained with (1) aceto-orcein, (2) G-banding, (3) R-banding, (4) $Q$-banding, (5) $C$-banding, (6) silver satellite staining.
Both parents, the two sibs, and the one half-sister (born in 1978) had normal karyotypes. One paternal uncle of the mother had 47,XY, trisomy 21 . No other abnormalities were reported in the family. The chromosomal abnormality in the proband is therefore probably de novo.

\section{Discussion}

A pericentric inversion in a chromosome 22 could have led directly to chromosome M2 in the proband. However, it is hard to explain how chromosome M1 originated in a conventional cytogenetic way. Such a long series of rare events as indicated in fig 3 seem too improbable to be a satisfactory explanation, and there is no support in published reports for a similar series of cytogenetic events. We must admit that we are unable to present a simple explanation to account for the distribution of bands and the size of chromosome M1. We feel, however, that it
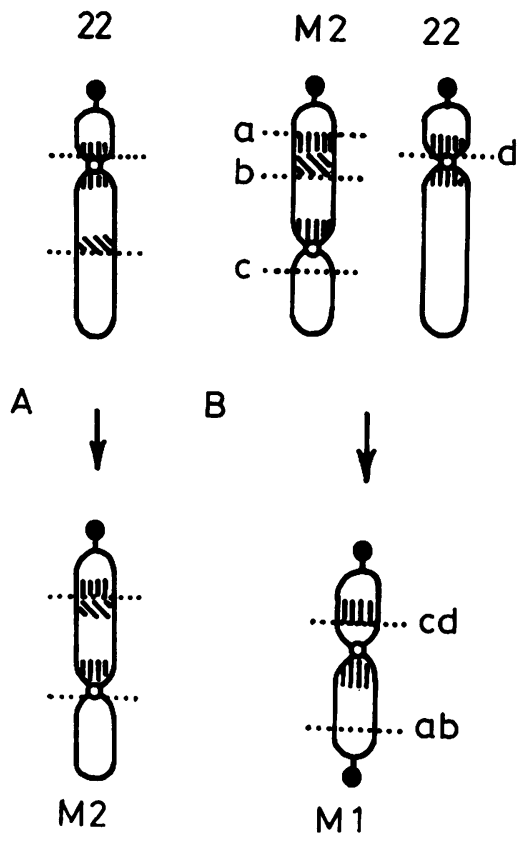

FIG 3 Origin of abnormal chromosomes through a series of breaks and exchanges. A. A pericentric inversion in one chromosome 22 results in M2. B. Three breaks in the inverted chromosome and one break in the normal 22 with deletion of the material between $a$ and $b$ and exchange $c-d$ result in chromosome $M 1$. The events in $B$ must have occurred during meiotic prophase since both $M 2$ and $M 1$ are preserved. Hatching: vertical, C-banding; diagonal, G-banding. 
is likely that the aberrations are the result of a major 'catastrophe' in meiosis in one of the parents, rather than a long series of independent aberrations.

According to our interpretation, two chromosomes 22 have participated in the formation of the marker chromosomes M1 and M2, so that the patient has chromosome 22 material partly in triplicate and partly in quadruplicate. At least one chromosome 22 must be involved, otherwise the patient would have monosomy 22, which as far as we know at present is not compatible with life. ${ }^{6}$

Most of the chromosome material that we assume that our patient has in quadruplicate consists of constitutive heterochromatin and satellites which are reported to be inactive. ${ }^{7}$ Therefore, the clinical manifestations should arise mainly from the partial trisomy for the long arm of 22. Certain features have been reported to be characteristic of partial trisomy $22 .{ }^{4}$ Our patient has some of these characteristics including mental retardation, growth retardation, antimongoloid slanting of the eyes, frontal bossing, broad nose, downturned mouth, micrognathia, large low-set ears with preauricular sinuses, and a striking resemblance to the patient described by Garlinger et al. ${ }^{4}$ Thus, these clinical features support our interpretation of the marker chromosomes in the patient.

I L HANSTeEn, L Schirmer, S Hestetun, AND A BRøGGER Laboratory of Genetics and Department of Paediatrics, Telemark Central Hospital, Porsgrunn; and Laboratory of Genetics, Norsk Hydro's Institute for Cancer Research, Oslo, Norway

\section{References}

1 Alfi OS, Sanger RG, Donnell GM. Trisomy 22: a clinically identifiable syndrome. Birth Defects 1975;XI:241-5.

2 Bofinger MK, Soukup SW. Cat eye syndrome. Am J Dis Child 1977;131:893-7.

8 Emanuel BS, Zackai EH, Aronson MM, Mellman WJ, Moorhead PS. Abnormal chromosome 22 and recurrence of trisomy-22 syndrome. J Med Genet 1976;13:501-6.

4 Garlinger P, McGeary SA, Magenis E. Partial trisomy 22: a recognizable syndrome. Clin Genet 1977;12:9-16.

5 Zellweger H, Ionasescu V, Simpson J, Burmeister L. The problem of trisomy 22. Clin Pediatr 1976;15:601-17.

- DeCicco F, Steele MW, Pan S, Park SC. Monosomy of chromosome no 22: a case report. J Pediatr 1973;83: 836-8.

7 Soudek D, Sroka H. C-bands in seven cases of accessory small chromosomes. Clin Genet 1977;12:285-9.

Requests for reprints to Dr Inger-Lise Hansteen, Laboratory of Genetics, Telemark Sentralsjukehus, Olavsgt 26, 3900 Porsgrunn, Norway.

\section{Concurrence of anorexia nervosa} and yellow mutant albinism

SUMMARY A review of published clinical reports $\frac{\overline{\bar{c}}}{\mathrm{~s}}$ shows that anorexia nervosa has been found in $\overrightarrow{\widetilde{\sigma}}$ association with several genetic anomalies, princi- $\stackrel{2}{v}$ pally gonosomal aneuploidy. An additional, and ${ }^{\infty}$ unique, association is described here: a case of $\vec{\circ}$ anorexia nervosa in a patient with the yellow $\vec{\omega}$ mutant form of oculocutaneous albinism and noo other apparent chromosomal abnormalities. While the concurrence of these two disorders in. a single person is apparently a chance pheno-.. menon, our review of experimental publications $\vec{\circ}$ shows that feeding disturbances also occur in yellow mutant mice. Such complementary? findings suggest the need for continuing investi- $\rightarrow$ gation of the genetic foundations of eating음 behaviour.

Anorexia nervosa is a feeding disorder occurring $\overrightarrow{0}$ primarily in adolescent women and is apparently. increasing in incidence. ${ }^{1}$ Primary symptoms include severe self-induced malnutrition with weight loss exceeding $25 \%$, distorted bodily perceptions, or implacable attitudes towards food, weight, and eating. These symptoms occur in the absence of ${ }_{\mathcal{Q}}^{\mathbb{D}}$ other physical or psychiatric illness that would $\underset{\rightarrow}{\rightarrow}$ account for this. ${ }^{2}$ Frequent secondary manifestations $\frac{}{3}$ include amenorrhoea, bradycardia, lanugo hair, $\supset$ hyperactivity, bulimia, and vomiting. Currento theories regarding the aetiology of anorexia nervosa postulate intrafamilial, psychodynamic, genetic, or hypothalamic factors. Although no genetic basis for:the disease has been clearly established, at least ten 3 . cases in association with gonosomal aneuploidyo (Turner's syndrome) have been reported since $19633^{3-13}$ Halmi and associates have argued per-o suasively that these cases reflect a much greater than chance concurrent incidence of $\mathrm{XO}$ gonosomeo abnormality and anorexia nervosa. ${ }^{56}$ On the other hand, Walinder and Mellbin ${ }^{14}$ found normalos karyotypes in all 30 anorexic patients they observed. N

Because of the epidemiological implications of an potential linkage between gonadal dysgenesis and $\omega$ anorexia nervosa, we have been conducting genetic gynaecological, and urological studies as part of the? medical work-up of anorexic patients receivingos treatment at University of Minnesota Hospitals during the past several years. Results of these studies will be reported elsewhere; to date they show noه significant incidence of sex chromosome anomalies in this patient population. One exceptional case, $\frac{\circ}{O}$ 